\title{
Variability of the Relationship between Electrophysiology and BOLD-fMRI across Cortical Regions in Humans
}

\author{
Christopher R. Conner, Timothy M. Ellmore, Thomas A. Pieters, Michael A. DiSano, and Nitin Tandon \\ Vivian L. Smith Department of Neurosurgery, University of Texas Medical School, Houston, Texas 77030
}

The relationship between blood oxygenation level-dependent (BOLD) functional MRI (fMRI) signal and the underlying neural electrical activity in humans is a topic of intense interest to systems neuroscience. This relationship has generally been assumed to be invariant regardless of the brain region and the cognitive task being studied. We critically evaluated these assumptions by comparing the BOLDfMRI response with local field potential (LFP) measurements during visually cued common noun and verb generation in 11 humans in whom 1210 subdural electrodes were implanted. As expected, power in the mid-gamma band $(60-120 \mathrm{~Hz})$ correlated positively $\left(r^{2}=\right.$ $\left.0.16, p<10^{-16}\right)$ and power in the beta band $(13-30 \mathrm{~Hz})$ correlated negatively $\left(r^{2}=0.09, p<10^{-16}\right)$ with the BOLD signal change. Beta and mid-gamma band activity independently explain different components of the observed BOLD signal. Importantly, we found that the location (i.e., lobe) of the recording site modulates the relationship between the electrocorticographic (ECoG) signal and the observed fMRI response $\left(p<10^{-16}, F_{21,1830}=52.7\right)$, while the type of language task does not. Across all brain regions, ECoG activity in the gamma and beta bands explains $22 \%$ of the fMRI response, but if the lobar location is considered, $28 \%$ of the variance can be explained. Further evaluation of this relationship at the level of individual gyri provides additional evidence of differences in the BOLD-LFP relationship by cortical locus. This spatial variability in the relationship between the fMRI signal and neural activity carries implications for modeling of the hemodynamic response function, an essential step for interregional fMRI comparisons.

\section{Introduction}

Functional magnetic resonance imaging (fMRI)-related techniques are widely applied to the study of human cognition (Indefrey and Levelt, 2004; Xue et al., 2010). An assumption underlying the analysis of these data is that the measured hemodynamic response provides spatially invariant information about neural activity driving the response. Prior evaluations of the relation between electrophysiologic activity and the hemodynamic response in sensory systems (Logothetis et al., 2001) have revealed that the blood oxygenation level-dependent (BOLD) signal correlates best with local field potentials (LFPs), less so with multiunit activity, and poorly with neuronal spiking. Given the spatial variability in the component processes contributing to the LFP, it is possible that there may be interregional variability in LFP-BOLD coupling (LBC) (Logothetis, 2008). Recent work using scalp EEG recordings in humans and across diverse re-

\footnotetext{
Received March 22, 2011; revised July 15, 2011; accepted July 21, 2011

Author contributions: N.T. designed research; C.R.C., T.M.E., T.A.P., M.A.D., and N.T. performed research; C.R.C. and N.T. contributed unpublished reagents/analytic tools; C.R.C. analyzed data; C.R.C. and N.T. wrote the paper.

The authors declare no competing financial interests.

This work was supported by the Center for Clinical and Translational Sciences, National Institutes of Health Clinical and Translational Award KL2 RR0224149 from the National Center for Research Resources and by the Vivian Smith Foundation for Neurologic Research. We thank Vips Patel for his help with MR scanning, the nurses and the EEG technicians at the epilepsy monitoring unit at Memorial Hermann Hospital for facilitating patient recordings, Jeremy Slater, Giridhar Kalamangalam, and Omotola Hope for contributing patients to the study, and Stephen Dreyer, Thomas $0^{\prime} \mathrm{Neill}$, and Mike Beauchamp for initial programming of electrode localization software, stimulus presentation software, and AFNI processing streams, respectively. We also thank Arne Ekstrom, Giridhar Kalamangalam, Valentin Dragoi, and Andreas Alexopoulos for thoughtful comments on this manuscript.

Correspondence should be addressed to Nitin Tandon, 6431 Fannin Street, Suite G.500, Houston, TX 77030. E-mail: nitin.tandon@uth.tmc.edu.

DOI:10.1523/JNEUROSCI.1457-11.2011

Copyright $\odot 2011$ the authors $\quad 0270-6474 / 11 / 3112855-11 \$ 15.00 / 0$
}

gions in animals (Martuzzi et al., 2009; Sloan et al., 2010) has specifically challenged the assumption of a stable LBC across brain regions. This makes it important to critically evaluate the assumption of spatially invariant coupling between the BOLD signal and direct measures of the underlying neural activity during cognitive processes.

Prior human studies that have evaluated the LBC have been limited to sensory or motor processes (Mukamel et al., 2005; Goense and Logothetis, 2008) or constrained to a particular brain region (Ekstrom, 2010; Ojemann et al., 2010; Scheeringa et al., 2011) or a specific frequency band. Analyses of sparse datasets have led others (Lachaux et al., 2007; Ekstrom, 2010) to propose that holistic, unbiased evaluations of the LBC across patients, brain regions, and paradigms be performed. Intracranial EEG recordings with implanted subdural electrodes (SDEs) are optimal for observing interactions in broadly disseminated cell assemblies, a salient feature required to make comparisons with whole brain fMRI measures. We recently evaluated the relationship between these measures during the delay period of a working memory task (Khursheed et al., 2011). Given the relatively weak changes from baseline seen in this task, only sites with activation during either electrocorticography (ECoG) or fMRI were compared. Therefore, few electrodes were used in this comparison $(n=118)$ with virtually none situated outside of frontal and temporal lobes, rendering meaningful interregional comparisons impossible. Furthermore, using only suprathreshold electrodes for comparisons might violate homoscedasticity assumptions in the regression models computed with these data.

To overcome the various limitations of prior studies, we studied 11 patients with refractory epilepsy who underwent preoperative whole brain BOLD-fMRI and subsequent ECoG recording 
Table 1. Numbers of trials and electrodes for the 11 patients used in the analysis

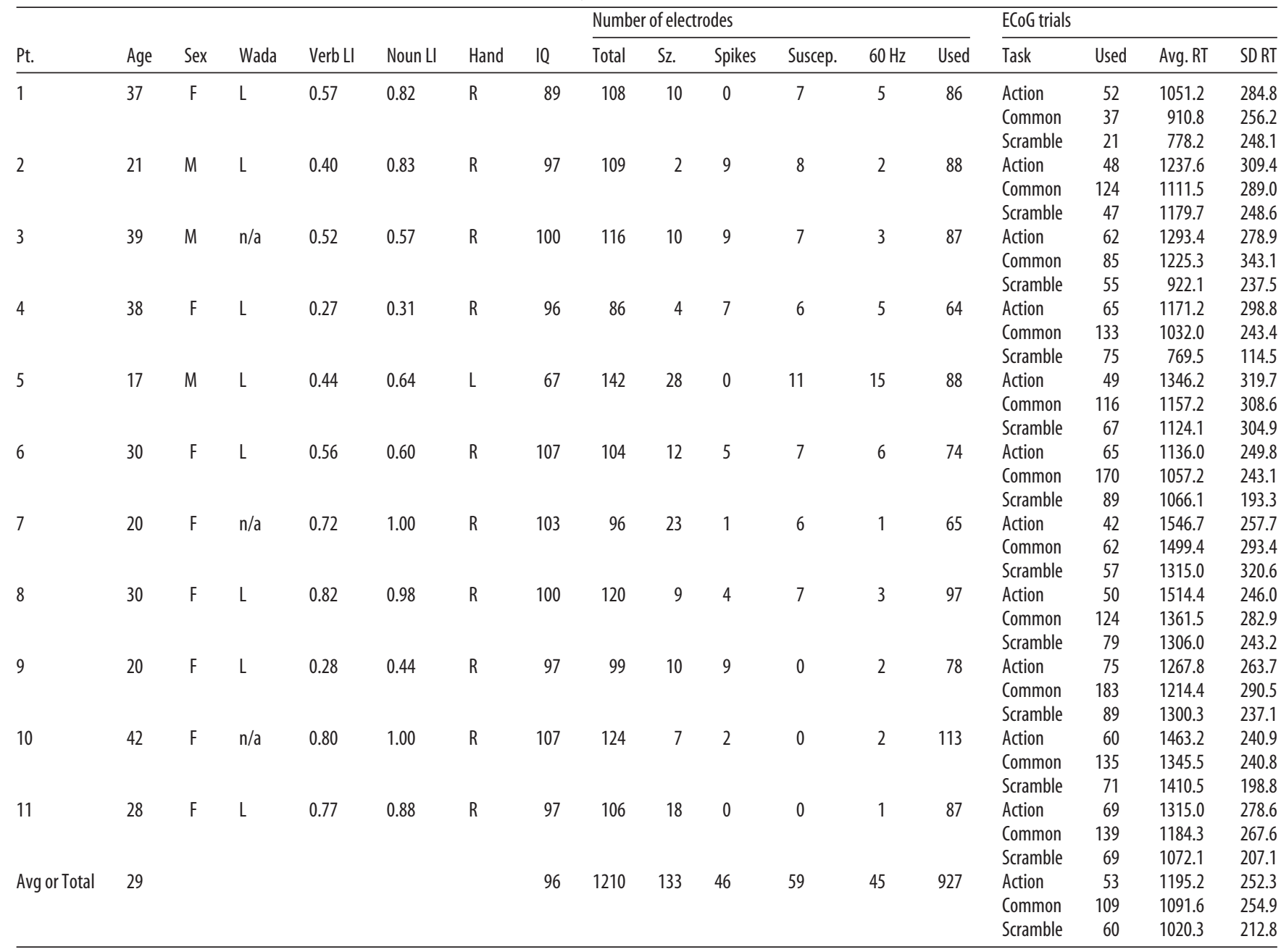

We sub-selected electrodes out of the total number of SDEs (Total) those that did not lie over overt seizure onset sites (Sz) or frequent generators of abnormal spikes, sampled cortex unaffected by susceptibility artifact on EPI (Suscep.), and were free of $60 \mathrm{~Hz}$ noise during ECoG recording sessions $(60 \mathrm{~Hz})$. After removing these electrodes, 927 electrodes (Used) across these patients were used for the correlations and the modeling. Patients (Pt.) were scored for accuracy during ECOG recording sessions and trials were excluded if they were incorrect or took too long to respond ( $>2 \mathrm{~s}$ ). The means and standard deviations of the reaction times were similar across patients. LI, Laterality index; $\mathrm{n} / \mathrm{a}$, not applicable; Avg, average.

from 1210 subdural electrodes over the language-dominant hemisphere. Patients performed similar, visually cued verb and noun generation tasks in both methodologies. These tasks are robustly performed by patients and activate overlapping, disseminated cortical substrates (Price and Friston, 1999; Indefrey and Levelt, 2004) consistently sampled by the typical SDE coverage in these patients (Tandon, 2008). Given the participation of both large- and small-scale networks along with processes involved with the inhibition of inappropriate responses (Liljestrom et al., 2009), these tasks seem well suited to evaluate the relationship between the underlying neural activity and how it drives the BOLD signal in disparate brain areas.

\section{Materials and Methods}

Eleven patients with medically refractory epilepsy (mean age 29 years, 8 females, 10 right-handers) were scheduled for intracranial EEG to localize seizures and enrolled in the study. Informed consent was obtained following study approval by our institution's committee for protection of human subjects. Functional and high-resolution anatomical magnetic resonance imaging data were acquired before electrode implantation. The time interval between MR scanning and LFP acquisition was 6-10 days. Eight of the eleven patients underwent intracarotid injection of sodium amytal (the Wada procedure) (Wada and Rasmussen, 2007) for lateralization of language function and were found to be left-hemisphere dominant (Table 1).

MR imaging. All participants were scanned using a 3T whole-body MR scanner (Philips Medical Systems) equipped with an eight-channel SENSE head coil. T1-weighted anatomical images were collected using a magnetization-prepared $180^{\circ}$ radiofrequency pulse and rapid gradientecho sequence with $1 \mathrm{~mm}^{3}$ voxels. Functional images were obtained with a gradient-recalled echo-planar imaging (EPI) sequence (Indefrey et al., 1997). Thirty-three axial slices ( $3 \mathrm{~mm}$ slice thickness, 2.75 in-plane resolution, TE $30 \mathrm{~ms}$, TR $2015 \mathrm{~ms}$, flip angle $90^{\circ}$ ) were collected during two visually cued covert language production tasks, common noun and verb generation. Stimuli were presented in a block design (Salmelin et al., 1994; Hamberger et al., 2005; Ellis et al., 2006; Specht et al., 2008). Prescan training was accomplished using similar, nonidentical stimuli. Each participant underwent scanning during two runs of each task (eight blocks per run, 136 TR volumes, $20 \mathrm{~s}$ of task, and $14 \mathrm{~s}$ of controlscrambled images). Data were thereby collected for 160 individual stimuli each for noun and verb generation and for 224 stimuli of scrambled images (112 during each naming task). During scanning, visual stimuli were presented at the onset of each functional image volume with Presentation software (version 11, Neurobehavioral Systems) using a screen positioned above the eyes (IFIS, Invivo). Each pictorial stimulus (task or scrambled) was on screen for $1500 \mathrm{~ms}$, with an interstimulus interval of $515 \mathrm{~ms}$ (Fig. 1A). During noun generation participants named the ob- 

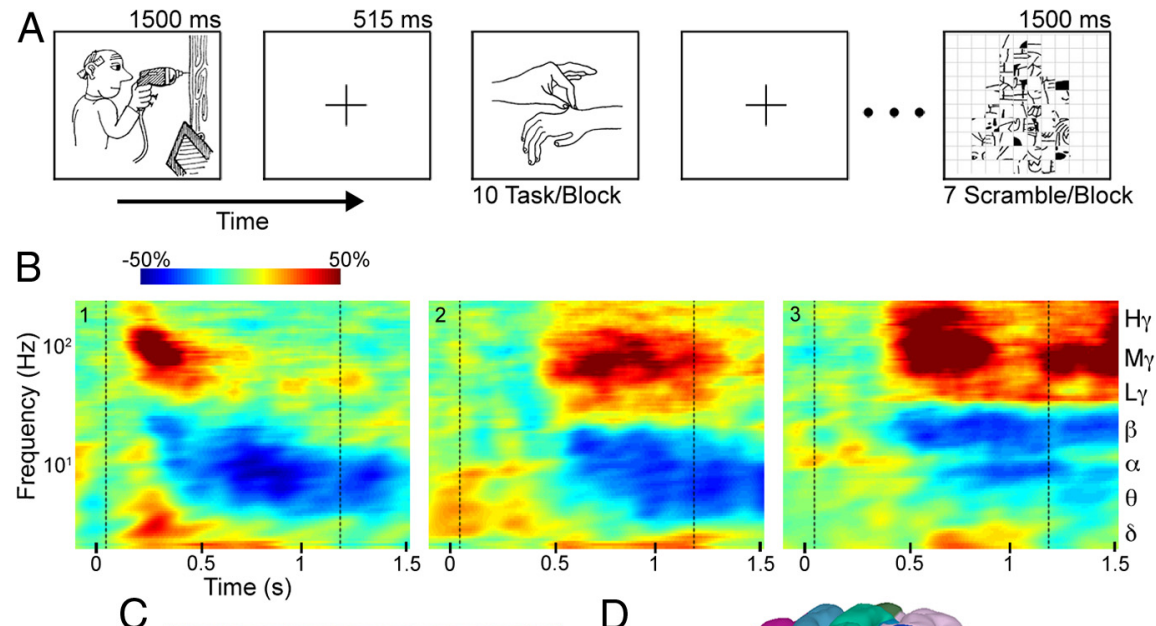

C
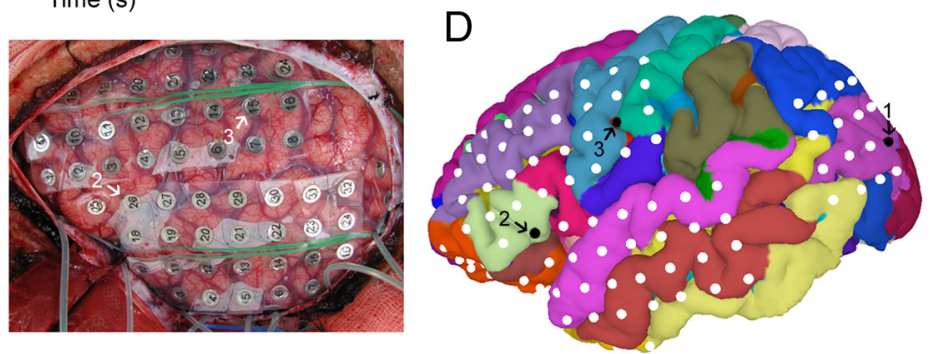

Figure 1. Representation of experimental paradigm and the spectral changes in the LFPs. $A$, Examples of visual stimuli for verb generation on screen for $1500 \mathrm{~ms}$ during both fMRI and ECOG acquisition. The TR during fMRI acquisition was $2015 \mathrm{~ms}$. Each fMRI block consisted of 10 images of verb or noun stimuli followed by 7 images of scrambled versions of the same stimuli. B. Spectrograms for a single subject computed using analytic signal processing. Spectrogram 1, V1; spectrogram 2, Broca's area (pars triangularis); spectrogram 3, M1 mouth. Spectral changes are depicted as percentage increases in power over a prestimulus baseline. The correlation between FMRI and LFP was carried out using $t$ statistics computed from the task vs the scrambled images condition. The time window between the vertical dotted lines in each graph, from $50 \mathrm{~ms}$ to mean reaction time minus one standard deviation, was averaged over trials to get the mean responses. $C$, Intraoperative photograph obtained after placement of subdural electrodes on the left hemisphere. $\boldsymbol{D}$, Representation of the same SDEs as spheres on a 3D automatically parcellated cortical surface generated using the same patient's MRI scan.

jects presented, and during verb naming they generated an action word; e.g., in regard to Figure $1 A$ they would generate verbs like "drilling" and "pinching." In each case they made no attempt at overt vocalization and pressed a button with the right thumb at the same time if they were successful. During the control condition, subjects viewed scrambled versions of the visual stimuli and thought of the word "scrambled" without overt vocalization while performing an alternate button press with the thumb (Ellmore et al., 2010). Patient responses were monitored in real time using a fiber optic response pad (fORP) connected to a fORP interface unit (Current Designs) and by video monitoring of the patients face using a closed circuit television.

Structural image processing, spatial transformations, functional image realignment, and statistical analyses were performed with AFNI (Cox, 1996). Each fMRI volume was aligned to the skull-stripped anatomical MRI using a registration algorithm with a mutual information cost function and bicubic resampling. The magnitude of each patient's translational and angular head movements was inspected by examining the output realignment parameters to exclude data corrupted by gross motion artifact. The aligned 4D dataset was spatially smoothed with a $3 \mathrm{~mm}$ Gaussian filter, and an omnibus $F$ ratio and corresponding probability value for each task versus control epoch was computed at each voxel time series by multiple regression. Lastly, a grouped analysis of the fMRI data for all 11 patients was carried out for each naming task. EPI datasets aligned to the anatomical MRI were transformed into Talairach space, and a grouped ANOVA was computed for each task versus scrambled condition.

To verify laterality in patients who did not have the Wada test, a laterality index was calculated using the language fMRI data (Ellmore et al., 2010). Masks of Brodmann areas 44 and 45 for each hemisphere were constructed using a standard atlas. The number of significant voxels $(p<0.001)$ during each task (noun and verb naming) versus the control (scrambled) condition was computed for each hemisphere. The laterality index was computed as equal to (\#L $-\# \mathrm{R}) /(\# \mathrm{~L}+\# \mathrm{R})$, where $\mathrm{L}$ is left hemisphere and right is right hemisphere. Positive values are associated with lateralization to the left hemisphere. All 11 patients were clearly left hemisphere lateralized for language function (Table 1).

Electrode placement and localization. The electrode localization methodology and recording strategies were similar to those previously described by our group during ECoG of other cognitive paradigms (Swann et al., 2009; Tertel et al., 2010; Khursheed et al., 2011). Briefly, subdural circular platinum-iridium electrodes with a top hat design $(4.5 \mathrm{~mm}$ total diameter, $3 \mathrm{~mm}$ contact with the cortex, 10 $\mathrm{mm}$ interelectrode distance, embedded in a silastic sheet from PMT Corporation) were implanted as clinically indicated using standard techniques (Tandon, 2008). Postoperative CT scans were obtained and coregistered with the preimplantation MRI. SDEs were localized on the CT scan and then, using custom in-house software, projected onto a cortical surface model generated by FreeSurfer (Dale et al., 1999) with in-house software and visualized in SUMA (Saad et al., 2003) as geometric spheres. These locations were manually verified and optimized as necessary using intraoperative photographs taken both at the time of SDE implant and explant (Fig. 1C). Electrodes were then given anatomical labels in semiautomated fashion, using a parcellation scheme (http:// surfer.nmr.mgh.harvard.edu/fswiki) generated by FreeSurfer (Fig. $1 D$ ). Lobar and gyral locations were confirmed visually by an expert in human neuroanatomy (N.T.). The lobar subgrouping included frontal, parietal, temporal, and occipital lobes. The gyral subgrouping was done under the following categories: superior frontal, middle frontal, Broca's area (pars opercularis, triangularis and orbitalis), orbitofrontal cortex, precentral, postcentral, inferior parietal (supramarginal, angular, and inferior parietal lobule), remaining parietal (superior parietal lobule and precuneus), superior temporal, middle temporal, inferior temporal, fusiform, and parahippocampal. Regions with sparse coverage (e.g., occipital lobe) were not subcategorized.

Electrocorticography. Patients were asked to perform the same naming tasks as they had during the functional imaging using similar stimuli. Recordings were carried out between 4 and $7 \mathrm{~d}$ after grid implantation. Stimuli were displayed on a 15 inch LCD screen positioned at eye level for $1500 \mathrm{~ms}$ using Presentation software. Concurrent audiovisual recordings were time locked to the continual ECoG, and transistor-transistor logic pulses were used to label stimulus onset and offset times on the ECoG. Interstimulus interval was 5000-7000 ms. Patients were instructed to name out loud the stimuli presented and respond to scramble trials by saying "scrambled." Response times were extracted for each trial using the audio trace time locked to the ECoG. Patients were scored for accuracy and only correct trials with response time of $<2 \mathrm{~s}$ were included in the analysis (corresponding to the inter-stimulus interval during fMRI acquisition). Any trial where any electrode of the implanted arrays showed interictal epileptiform discharges (IEDs) was discarded from further analysis. All patients were presented with $>50$ trials of both noun and verb naming.

ECoG data were collected at $1000 \mathrm{~Hz}$ during naming using Nihon Kohden NeuroFax software (bandwidth $0.15-300 \mathrm{~Hz}$ ). Electrodes were 


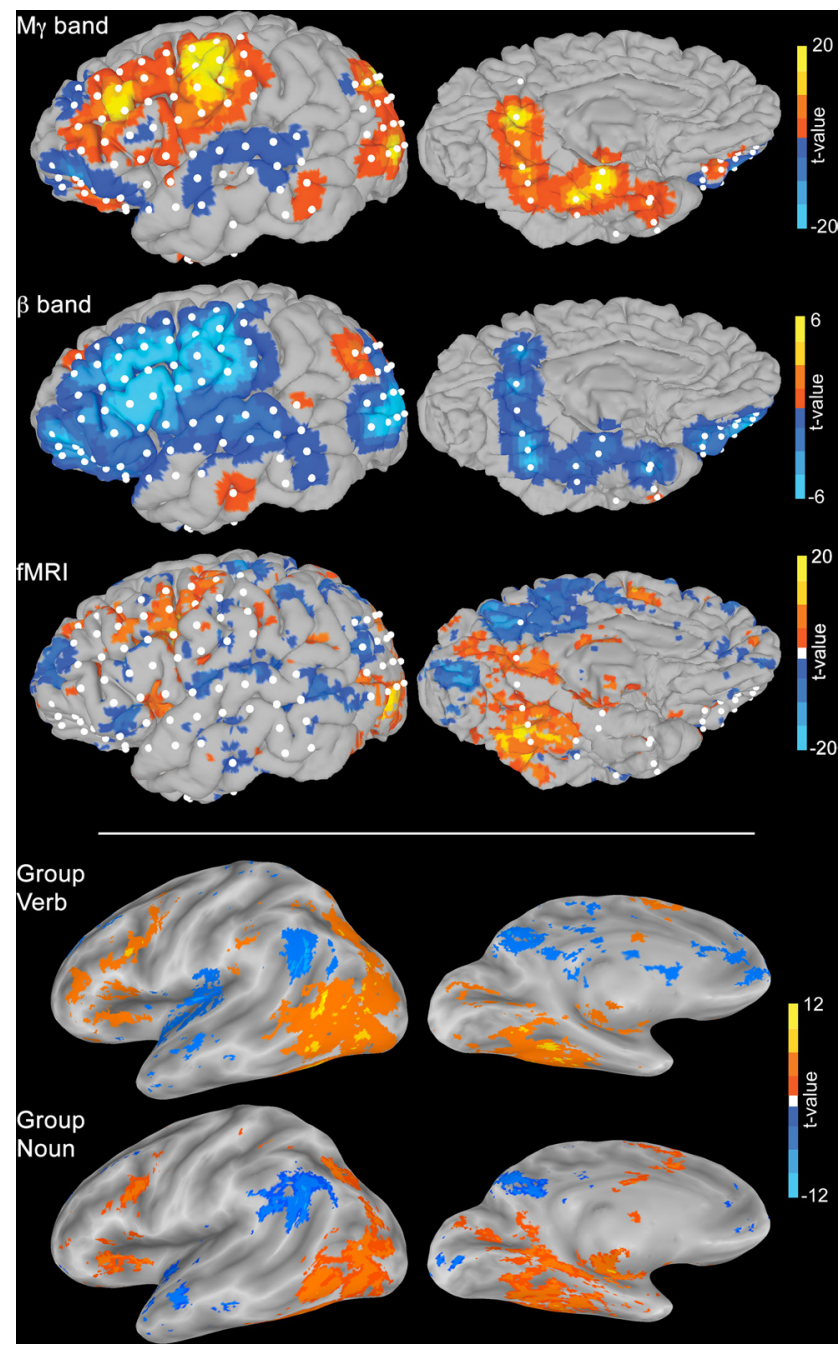

Figure 2. Representation of cortical activity during naming tasks versus viewing scrambled images. The top three rows represent data from a single illustrative patient during visually cued noun generation contrasted with scrambled images. Activity measured by ECOG in the midgamma (M $\gamma)$ band $(60-120 \mathrm{~Hz}$, top row) and the beta $(\beta)$ band ( $13-30 \mathrm{~Hz}$, second row) and by BOLD-fMRI (third row) is represented on the cortical surface to allow for direct visual comparison. The fMRI data shown here were unconstrained by the $8 \mathrm{~mm}$ V0ls placed around each electrode in the analysis to give a complete representation of all activation. While unthresholded fMRI and LFP data were used in the correlation, for illustrative purposes this fMRI dataset is thresholded at $p<0.001$. The lower two rows depict fMRI analysis for the entire group ( $n=$ 11 ) displayed on the inflated gray-white junction for verb (fourth row) and noun (fifth row) generation $(p<0.01)$.

referenced to a common average of all electrodes except for those with 60 $\mathrm{Hz}$ noise or epileptiform activity when referenced to an artificial $0 \mathrm{~V}$ (Crone et al., 2001; Brown et al., 2008). The data were imported into MATLAB (MathWorks), and the patients' articulation times were extracted using the time-locked audio-video recording. To avoid any abnormal brain regions in the correlation analysis, all electrodes that showed any interictal activity or early involvement with seizure onsets were excluded. All electrodes with $>10 \mathrm{~dB}$ of noise in the $60 \mathrm{~Hz}$ band were also excluded. An important consideration was to systematically eliminate electrodes that lay over regions affected by susceptibility artifact during EPI imaging. To do this, the locations of all electrodes for each individual were visualized on the surface of the brain while simultaneously viewing fMRI activity (Fig. 2). To determine which regions were affected by the artifact, a mask was generated during processing that encompasses all data within the brain not affected by susceptibility artifact. Electrodes that lay outside this region were excluded accordingly (Table 1).
For the correlation, the ECoG spectral analysis was carried out independently using both Hilbert and Fourier transform techniques for the entire data analysis (the results were found to be generally similar and served to cross validate the entire analytic stream). The Hilbert transform (Le Van Quyen et al., 2001; Bruns, 2004) provided greater resolution in the temporal domain and allowed for precise bandpass filtering. It is therefore the analysis used to report results here and to generate the statistical and graphical representations of data. Analytic signal analysis was carried out by initially bandpass filtering (IIR Elliptical Filter, 10th order, $30 \mathrm{~dB}$ sidelobe attenuation, $0.5 \mathrm{~dB}$ passband ripple) the raw data into the seven bands: delta $(0-4 \mathrm{~Hz})$, theta $(4-8 \mathrm{~Hz})$, alpha $(8-13 \mathrm{~Hz})$, beta $(13-30 \mathrm{~Hz})$, low-gamma $(30-60 \mathrm{~Hz})$, mid-gamma $(60-120 \mathrm{~Hz})$, and high-gamma $(120-240 \mathrm{~Hz})$. A Hilbert transform was then used to obtain the analytic signal. The amplitude of the transform was smoothed (Savitzky-Golay FIR smoothing filter, 2nd order, frame length of 255 samples) and then averaged from $50 \mathrm{~ms}$ after stimulus onset to mean reaction time minus $1 \mathrm{SD}$ to compute the response in each band. A second set was constructed by using 50 bins uniformly centered on a logarithmic scale from 2 to $240 \mathrm{~Hz}$ with a logarithmic bandwidth ranging from 4 to 40 $\mathrm{Hz}$. In other words, the first bin extended from 0 to $4 \mathrm{~Hz}$ and the last from 220 to $260 \mathrm{~Hz}$. Response was measured using the $t$ value from a twosample unpaired $t$ test (given unequal trials in the two conditions) and assuming unequal variances between each task condition and the control (scrambled images) conditions.

Parameter selection. To compare the ECoG activity with the fMRI signal, spherical volumes of interest (VOIs) ranging from 5 to $15 \mathrm{~mm}$ were initially used to sample the fMRI. Across these radii, the LBC profiles were not significantly different, but the correlation was maximal for the 8 $\mathrm{mm}$ VOI. This VOI size was therefore chosen as the size used in the analysis. The VOIs were placed on the non-resampled fMRI datasets bounded by the cortical ribbon to eliminate voxels in white matter and those outside the brain. A voxel was considered within the VOI if at least $50 \%$ of it lay within the VOI. The average $t$ value of the fMRI for all voxels within the VOI was computed and imported into MATLAB. Overlays of the ECoG activity in each band were generated using a 3D Gaussian filter $(\mathrm{SD}=5 \mathrm{~mm})$ were constructed in MATLAB and represented on the pial surface (Lachaux et al., 2003) using SUMA (Fig. 2).

Regression analysis. Activity in each of the seven bands in each individual during both naming tasks was then regressed with the mean $t$ value of the BOLD signal change within the VOI placed around each electrode. Next, to elaborate the correlation values at a finer frequency resolution for the spectrum, activity estimates in logarithmically spaced bins were regressed with the BOLD signal change. A correlation coefficient and corresponding $p$ value were computed for each bin. Strictly speaking, this method is statistically suboptimal, as adjoining bins overlap and the data are not completely independent. Nevertheless, this analysis estimates the curve for correlation of the entire spectrum with the BOLD signal.

To precisely evaluate the null hypothesis that the LBC is spatially invariant, we modeled the observed BOLD signal. The variables used in this model were the activity in each of the seven ECoG bands and the contrast variables indicating individual patient identity, lobar locus of the recording electrode, and the experimental condition. In this regression, $y_{i t}$ is the average $t$ statistic in the VOI around the $i$-th electrode during the $t$-th task, $\beta_{\mathrm{o}}$ is the intercept, $\beta_{\mathrm{k}}$ is the regression coefficient for the $k$-th LFP band, $x_{\mathrm{kit}}$ is the $t$ statistic in that band, $l_{\mathrm{i}}$ is the linear contrast variable for the lobar location of that electrode (classified as occipital, frontal, parietal, or temporal), $s_{\mathrm{i}}$ represents which patient the electrode is from, and $T_{\mathrm{t}}$ is the contrast for the tasks. The remaining terms, $\beta_{\mathrm{l}}, \beta_{\mathrm{s}}$, and $\beta_{\mathrm{T}}$, are the regression coefficients for locus, subject, and task, respectively.

$$
y_{\mathrm{it}}=\beta_{\mathrm{o}}+\sum_{k=1} \beta_{\mathrm{k}} x_{\mathrm{kit}}+\beta_{\mathrm{l}} l_{\mathrm{i}}+\beta_{\mathrm{s}} s_{\mathrm{i}}+\beta_{\mathrm{T}} T_{\mathrm{t}}+\epsilon .
$$

To evaluate the LFP-BOLD coupling function further, the analytic process described above was repeated for data categorized by lobe. Confidence intervals (99\% uncorrected) around the Pearson's $r$ were calculated with a Fisher's $z$ transformation. The LBC function for each lobe was plotted alongside the results from the entire set of electrodes to 
highlight differences between each lobe and the mean. Additionally, scatter plots of the power in the each of the seven canonical bands versus the fMRI activity were generated. Estimates of divergence in the coefficients of the regression for each lobe were computed by dividing the differences between coefficients by their standard error (Paternoster et al., 1998). To investigate differences at finer spatial resolution than the level of a lobe, comparisons of activity at electrodes grouped by gyrus (identified using a FreeSurfer parcellation scheme; see above, Electrode placement and localization) were made. LBC curves and confidence intervals for all gyri with $>20$ electrodes were made and compared with all electrodes as was done by lobe.

\section{Results}

In all 11 patients, fMRI activations and event-related spectral changes were obvious over areas expected to be active in visual naming - primary visual cortex, fusiform gyrus, lateral occipital cortex, Broca's area, premotor cortex, and prefrontal cortex (Price et al., 1996; Price, 2000; Shapiro et al., 2006) (as expected, little lateral temporal activation was noted during these visually cued naming processes; Fig. 2). ECoG changes were most pronounced as increases in power at high frequencies (mid and high gamma) and decreases in power in low frequencies (alpha and beta) (Fig. 2). Of the total of 1210 electrodes implanted, 283 electrodes were excluded from the analysis as they overlay electrically abnormal cortex or had excessive radio frequency noise or overlay regions where there was EPI susceptibility artifact during the fMRI acquisition (See Table 1). The comparison between fMRI and LFP signals was made for the remaining 927 electrodes. Of these, 454 electrodes were over the frontal lobe, 265 were over the temporal lobe, 157 were over the parietal lobe, and 51 lay over the occipital lobe.

While patient performance was directly observable in the ECoG environment, performance in the scanner could only be inferred indirectly. Prior studies of naming have shown that the reaction times for verb generation are longer than those for noun generation. As expected (Szekely et al., 2005), reaction times (RTs) were significantly shorter for common noun generation than for verb generation in both experimental conditions (fMRI and ECoG). In the MR scanner the mean RT for verb generation was $1012 \mathrm{~ms}(\mathrm{SD}=288 \mathrm{~ms})$ and for noun generation it was 950 $\mathrm{ms}(\mathrm{SD}=293 \mathrm{~ms})$, which were significantly different for the group $\left(p<10^{-10}\right)$ indicating that patients were indeed performing the task adequately. The distinction between these RTs was in the same direction during ECoG recordings: verb, $1319 \mathrm{~ms}$ (SD = $335 \mathrm{~ms}$ ); noun, $1264 \mathrm{~ms}$ (SD = $371 \mathrm{~ms}$ ) (Table 1); also significantly different with $p<0.01$. Reaction times during LFP recordings were longer than those in the fMRI scanner as patients articulate their responses. The window over which the LFP changes are considered was tailored to both the subject and condition by adjusting the length of the epoch. For each individual (patient) and condition (verb, noun, and scramble images), a mean and SD were computed. The width of the LFP epoch was set from stimulus onset to mean articulation minus 1 SD. This reduced the effect of premotor and motor responses related to articulation in the ECoG condition. Paradigmatic differences were further minimized by use of a contrast condition of viewing scrambled images (which also included a button press in fMRI and said "scrambled" during ECoG).

\section{Regression}

Independent correlation estimates between the fMRI signal and ECoG activity in each of the narrow frequency bins revealed a strong negative correlation at about $20 \mathrm{~Hz}$ and a strong positive correlation at $90-100 \mathrm{~Hz}$ (Fig. 3). When the same estimate was
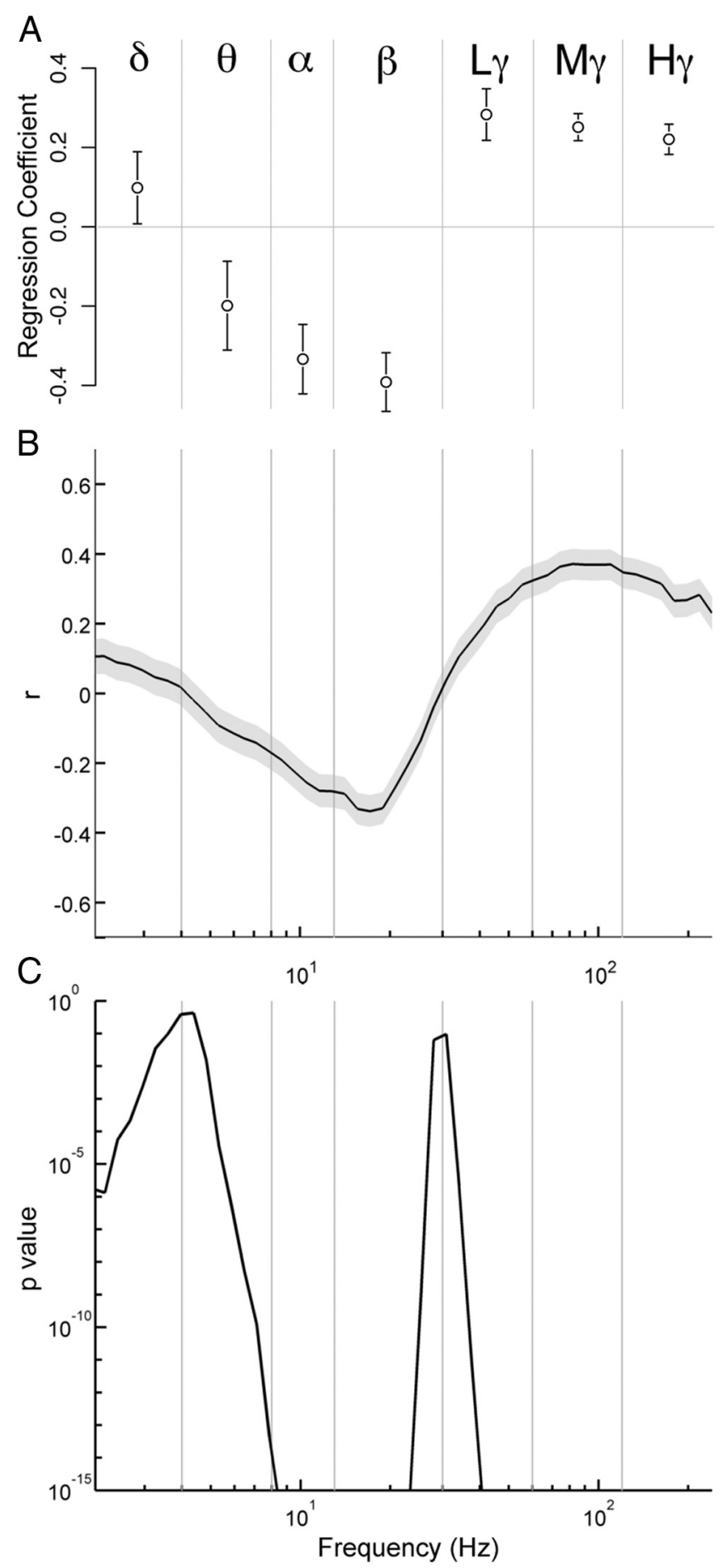

Figure 3. Correlation between the BOLD signal and the ECOG across brain regions. Data are from all 11 individuals ( $n=1210$ electrodes) during both naming tasks. Electrodes with electrical artifact and those overlying abnormal cortex or brain regions affected by susceptibility artifact during EPI acquisition were excluded. The total number of electrodes sites used in this comparison was 1853 ( 926 during noun and 927 during verb generation respectively). Neither fMRI nor ECoG datasets were thresholded before correlation. An $8 \mathrm{~mm}$ VOI around each ECOG electrode was used to sample the fMRI data. $A$, Regression coefficients with $99 \% \mathrm{Cls}$ between ECOG activity in each canonical band. B, C, Pearson's $r$ values with $99 \% \mathrm{Cl}$ (computed using Fisher's $z$ statistic) $(\boldsymbol{B})$ and the associated $p$ values $(\boldsymbol{C})$, analyzed using 50 frequency bins on a logarithmic scale from 2 to $240 \mathrm{~Hz}$, with logarithmic width from 4 to $40 \mathrm{~Hz}$. The inverse correlation at low frequencies (alpha and beta bands) inflects to a positive correlation at about $30 \mathrm{~Hz}$ (start of the gamma band). Different correlation values are noted in the low- ( $\mathrm{L}$; $30-60 \mathrm{~Hz})$, mid- $(\mathrm{M} \gamma ; 60-120)$, and high-gamma $(\mathrm{H} \gamma ; 120-240)$ bands with a peak around $90-100 \mathrm{~Hz}$. 
made with the seven canonical frequency bands, the strongest positive correlation (also the largest coefficient) was with the mid-gamma band $\left(r^{2}=0.16\right.$; coefficient $\left.=0.25\right)$. The strongest negative correlation was with the beta band $\left(r^{2}=0.09\right.$; coefficient $=-0.39$ ). We also evaluated the correlation between activity in the mid-gamma band and that in the beta band and found that they were very poorly correlated $\left(r^{2}=0.03\right)$ (Scheeringa et al., 2011).

An ANCOVA and a linear regression were computed using the seven frequency bands along with linear contrast variables for recording site (lobe), patient, and language task (verb or noun generation) task. This revealed a strong negative correlation in the beta band (coefficient $=-0.39, t$ value $=-8.44, F_{1,1830}=$ 139.7, $p<10^{-16}$ ), a strong positive correlation in the midgamma band $\left(\right.$ coefficient $=0.21, t$-value $=16.74, F_{1,1830}=327.0$, $\left.p<10^{-16}\right)$, a significant effect of lobe $\left(F_{3,1830}=52.7, p<10^{-16}\right)$, and significant although weaker effect of the individual patient $\left(F_{10,1830}=10.8, p<10^{-16}\right)$. The overall adjusted $r^{2}$ for this model was $0.32\left(F_{21,1830}=43.4, p<10^{-16}\right)$. The other regressors (remaining five bands and the task condition) were not significant. Model reduction was then performed to include only beta and mid-gamma bands as regressors with the fMRI activity. The adjusted $r^{2}$ for this second model was $0.22(F=259.4, p<$ $10^{-16}$ ). If information about the lobe was then added as a third regressor, the overall $r^{2}$ rose to 0.28 .

To evaluate the distinctions in the LBC further, we regressed the ECoG activity for each lobe in each of the seven bands with the corresponding BOLD signal change. Significance levels for divergence in the coefficients of the regression for each lobe (Paternoster et al., 1998) (Fig. 4) and for the correlation values (Cohen and Cohen, 1983) were both computed (Fig. 5). While the general pattern of low frequency-negative correlation and high frequencypositive correlation was conserved, there were notable distinctions in the LBC relationship across lobes. In the alpha and beta bands, a negative correlation was seen for all bands but was significantly smaller $(p<0.001)$ in frontal cortex. At higher frequencies (gamma), the regression coefficients were relatively invariant across brain locations while the values of the correlation were much smaller $(p<0.001)$ in the parietal lobe than in the rest of cortex. In the delta band, a positive correlation that was significantly $(p<0.01)$ different from that in the other lobes was present in the occipital lobe (Figs. 4). The relationship between high-frequency activity in occipital cortex with delta band activity has been suggested recently (Nagasawa et al., 2011) and may also be a ECoG correlate of the P100 response. To depict these distinctions visually, we also used the finer scaled logarithmically spaced bins to again obtain LBC response curves for each lobe, along with the $95 \%$ confidence bounds for the Pearson's $r$ values (Fig. 5).

Spatial variability in the $\mathrm{LBC}$ was then further evaluated at the sublobar level. Spatial locations were classified into 13 gyral locations using FreeSurfer. Differences in the correlations at each of these gyri were computed and significant and distinct deviations from the population were noted in several regions (Fig. 6). Dramatically distinct LBC relationships were seen in the superior temporal gyrus (STG) and middle temporal gyrus (MTG) and in the postcentral gyrus (PoCG). In all of these regions, little to no correlation between the fMRI signal with gamma band was noted, Although the negative correlation with the beta band was maintained in MTG and PoCG but not in STG. Conversely, the orbital cortex showed an almost complete absence of a negative correlation in the beta band; however, the gamma band relationship with the BOLD signal was maintained. Less striking but still
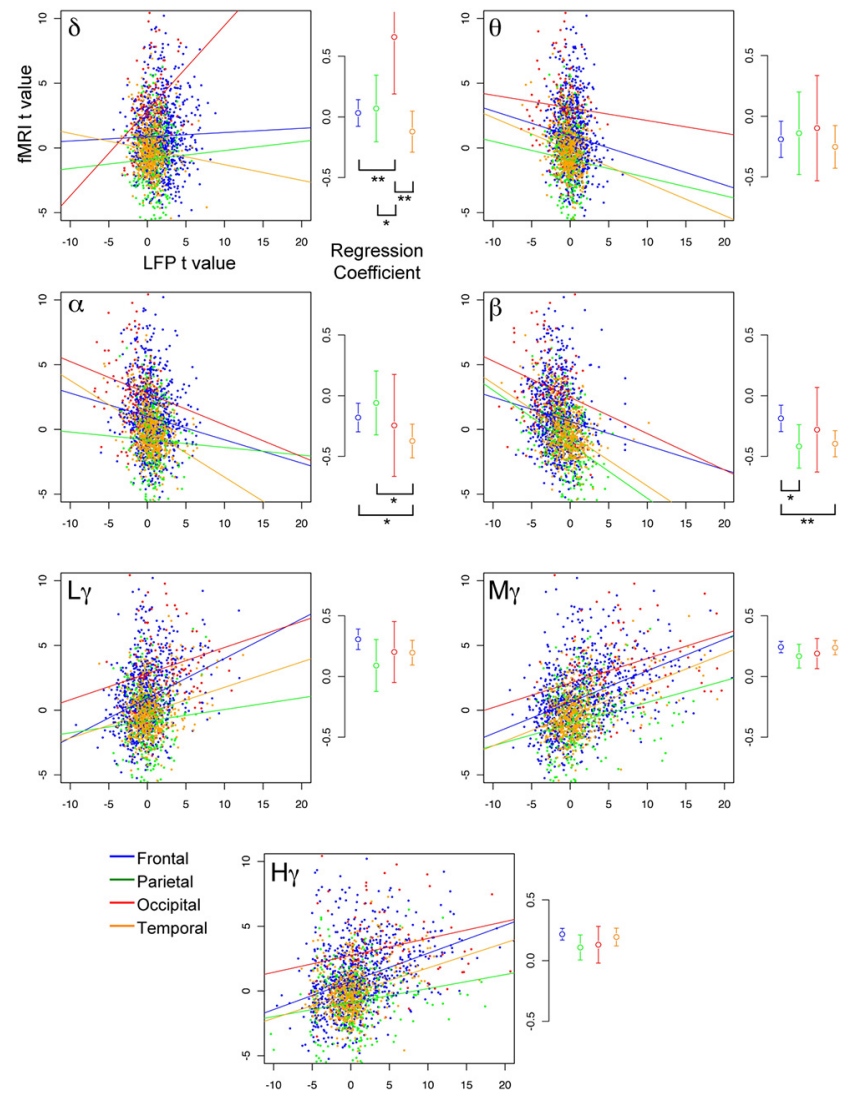

Figure 4. Correlations for each frequency band separated by lobe. Scatter plots of the data used in the lobe-specific regressions (Fig. 5) illustrate the differences between correlations across lobes. LFP power is plotted against fMRI power for each of the seven bands used in the comparison. Linear models fitted using each of frontal (blue), parietal (green), occipital (red), and temporal (orange) separately show significant variation in regression of LFP band power on fMRI-BOLD response at low frequencies. Regression coefficients (the slope of the regression line; Fig. 3A) for each band (along with $99 \% \mathrm{Cl}$, uncorrected) show that direction, magnitude, and variability are all location and band specific. Comparisons of the LBC function for each lobe against the others were performed to test for significant differences $\left({ }^{*} p<0.01\right.$, $\left.{ }^{* *} p<0.001\right)$. Significant differences were noted in low-frequency bands (delta, alpha, beta) but not in their higher-frequency counterparts (gamma). Differences were present in the beta band despite the fact that it was a significant regressor in the final linear model (see Results), further supporting the differences in the $\mathrm{LBC}$ function across lobes.

significant differences in the LBC function were noted in the precentral gyrus (positive correlation in delta and theta, less negative correlation in beta, and less positive correlation in gamma) and the middle frontal gyrus (less negative correlation in alpha).

Most cognitive fMRI studies report data only above an arbitrary statistical threshold. A problem with making comparisons between thresholded fMRI and the ECoG data is that thresholding may violate assumptions of normality and homoscedasticity in the data. Even so, we also computed a regression using thresholded fMRI data $(t>3.29$ or $t<-3.29)$ (data not shown) and found correlations across brain regions notably similar to the ones reported here. Specifically, this regression revealed a significant effect of lobar location $\left(F=28.3, p<10^{-16}\right)$ on the LBC function. One explanation of the spatial variance in the LBC function might be that nonlinearity could occur at the extremes of the HRF. Our analyses with thresholded data also show that if the correlation is performed for either fMRI activations or deactivations alone, the net result is not much different. Additionally, all patients underwent tailored cortical stimulation mapping (CSM) using standard techniques to identify essential language 

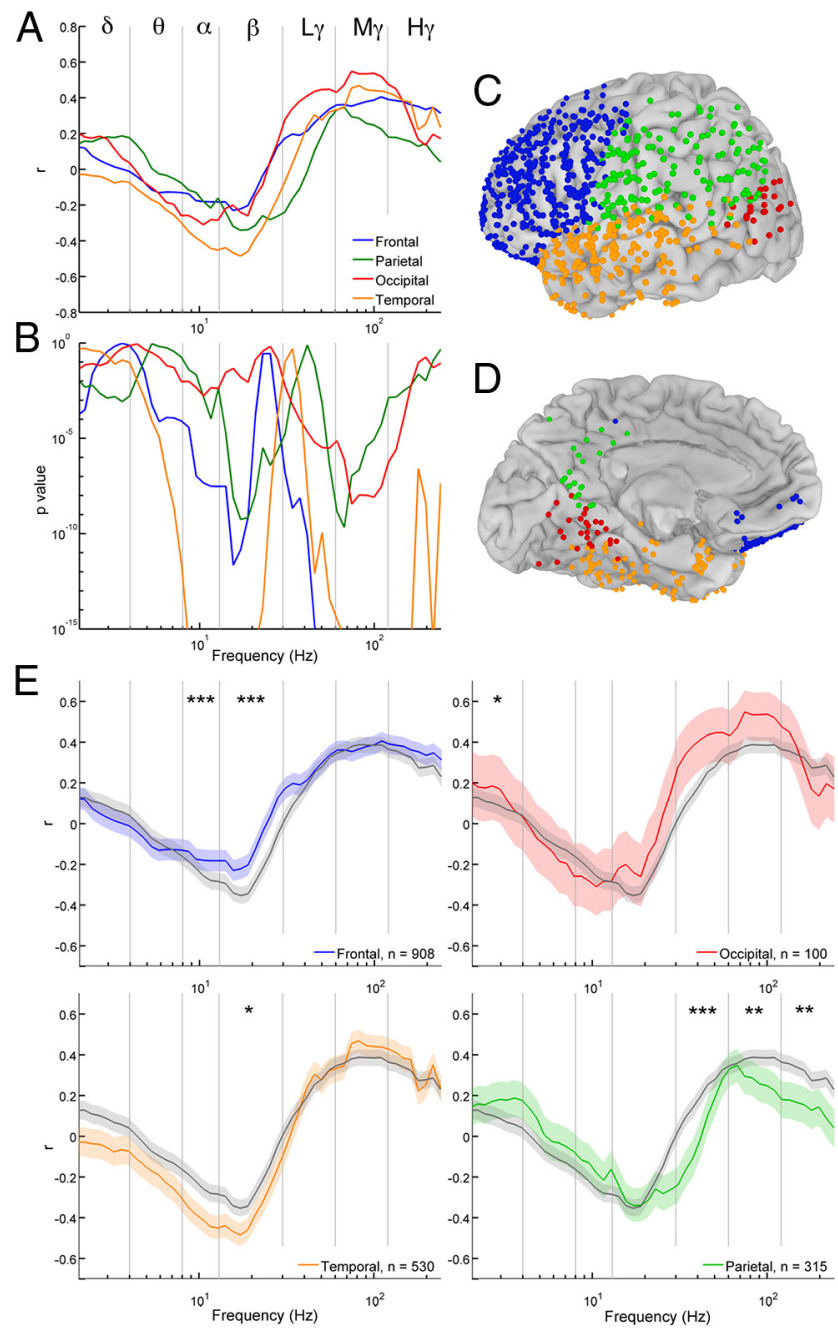

Figure 5. Effects of location on the correlation between the local field potential and the fMRI signal change. Data from both tasks (noun and verb naming) were pooled together as in Figure 3. $\boldsymbol{A}, \boldsymbol{B}$, Pearson's $r(\boldsymbol{A})$ and $p(\boldsymbol{B})$ values at each frequency bin are computed as a function of lobar location of the recording electrode. Significance of correlation was strongly affected by numbers of electrodes; therefore, lobes with the greatest numbers of samples (frontal and temporal) had the highest levels of significance. $\boldsymbol{C}, \mathbf{D}$, Locations and distributions of electrodes are shown colocalized on a single brain surface to which they were registered using a 12 parameter affine transform. The number of electrodes included in the analysis varied slightly between the two tasks (due to slight variations in noisy channels between the recording sessions for each task). In both tasks there were 454 electrodes over the frontal lobe and 265 over the temporal lobe. During verb naming there were 157 electrodes over the parietal lobe and 51 over the occipital lobe, while in common naming there were 158 and 49, respectively. $\boldsymbol{E}$, The mean correlation values for each lobe with $95 \%$ Cls are plotted. Mean response with $95 \%$ Cls across all electrodes is plotted in gray. Significance levels: ${ }^{*} p<0.01,{ }^{* *} p<0.001,{ }^{* * *} p<0.00001$.

sites (ELSs) for clinical purposes (Tandon, 2008). ELSs were identified in all cases, further proving that the left hemisphere was indeed the language-dominant hemisphere in these cases. These CSM results provided another way to threshold functional regions by significance. The majority of ELS were identified in Broca's area (25 sites), STG (40 sites), and MTG (10 sites). The LBC function for these CSM-positive sites were computed for each gyral subregion and compared with the CSM-negative sites in the same subregion. There were too few sites identified in other gyral locations to make meaningful comparisons. Significant differences between the CSM-positive sites and negative sites in each gyral region were noted in the gamma bands (Fig. 7).
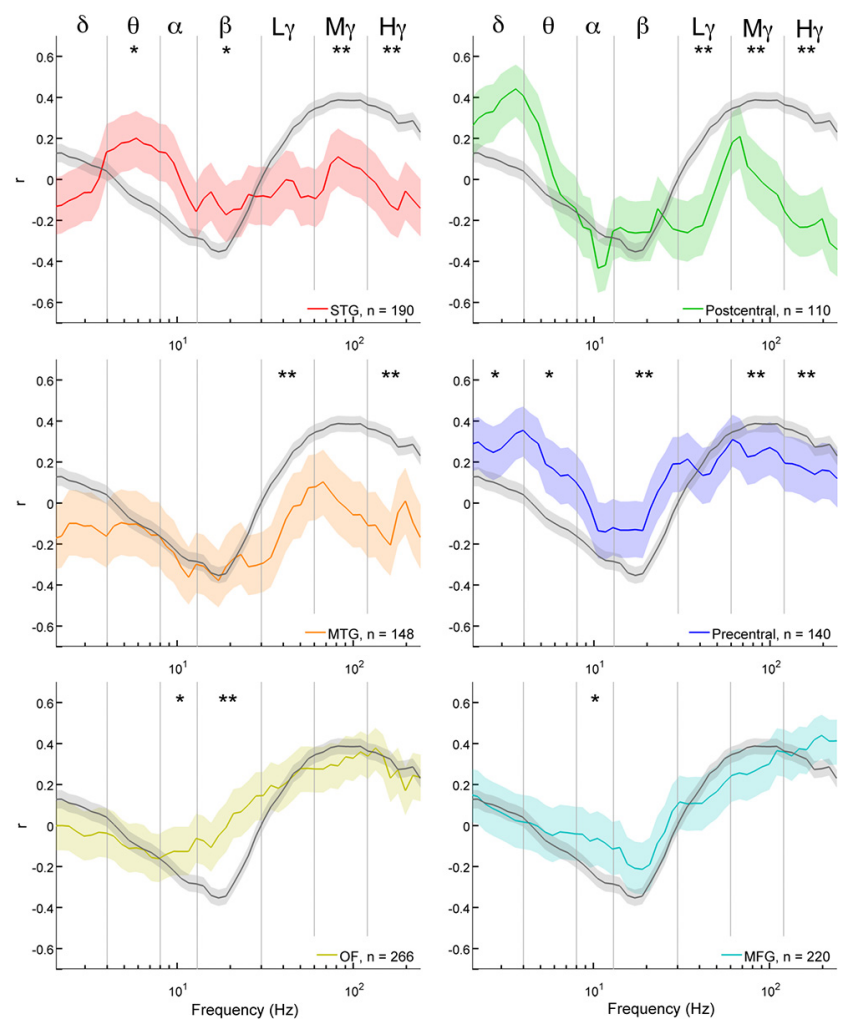

Figure 6. Variance in the function of LFP-BOLD coupling, LBC, across specific gyri. Total numbers of electrodes contributing to each group are shown in each graph. The gray line represents the correlation for all electrodes used in the analysis (Fig. 3B), while each of the colored lines contain only those electrodes at a specific gyrus. The shaded error bars around both lines reflect an uncorrected $p<0.05$. Significant deviations in correlation from the group are noted in STG, postcentral gyrus, MTG, precentral gyrus, orbitofrontal cortex (OF), and middle frontal gyrus (MFG). Differences in the $L B C$ relationship occur in all seven frequency bands. Significance levels: ${ }^{*} p<0.01,{ }^{* *} p<0.001$.

\section{Mixed effects model}

In the model generated above, the effects of individual variability were modeled as a linear contrast variable. Strictly speaking, variations in individual data contribute in a more unpredictable fashion than can be modeled linearly and are best categorized as a random effect. A mixed effects model (fitted in R using NLME package, version 3.1) was therefore generated. The fixed effects (subject invariant) were activity in the mid-gamma and beta bands and locus of the recording electrode. The random effects were subject-specific deviations from the fixed portions of the model. For the linear mixed effects model we selected the frontal lobe as the base locus because the largest number of electrodes were implanted there. The other three lobes were compared with this base locus. The model revealed significant effects of activity in the beta band (coefficient $=-0.23, t$ value $=-8.50, \mathrm{df}=$ $\left.1836, p<10^{-16}\right)$, the mid-gamma band $(0.21, t$ value $=16.72$, $\left.p<10^{-16}\right)$, and locus $\left(F_{3,1836}=55.1, p<10^{-16}\right)$. For the random effects (subject) variable, the variance was 2.21 (95\% CI, 2.14-2.28). The results of this model were similar to those of general linear models performed earlier, suggesting that these two bands plus the location of the recording electrode do contribute meaningfully to the observed BOLD signal change.

\section{Discussion}

This work reveals that the LFP-BOLD relationship is best described by activity in two bands- beta and mid-gamma. Activity in these bands can model $\sim 22 \%$ of the variance in the fMRI signal 

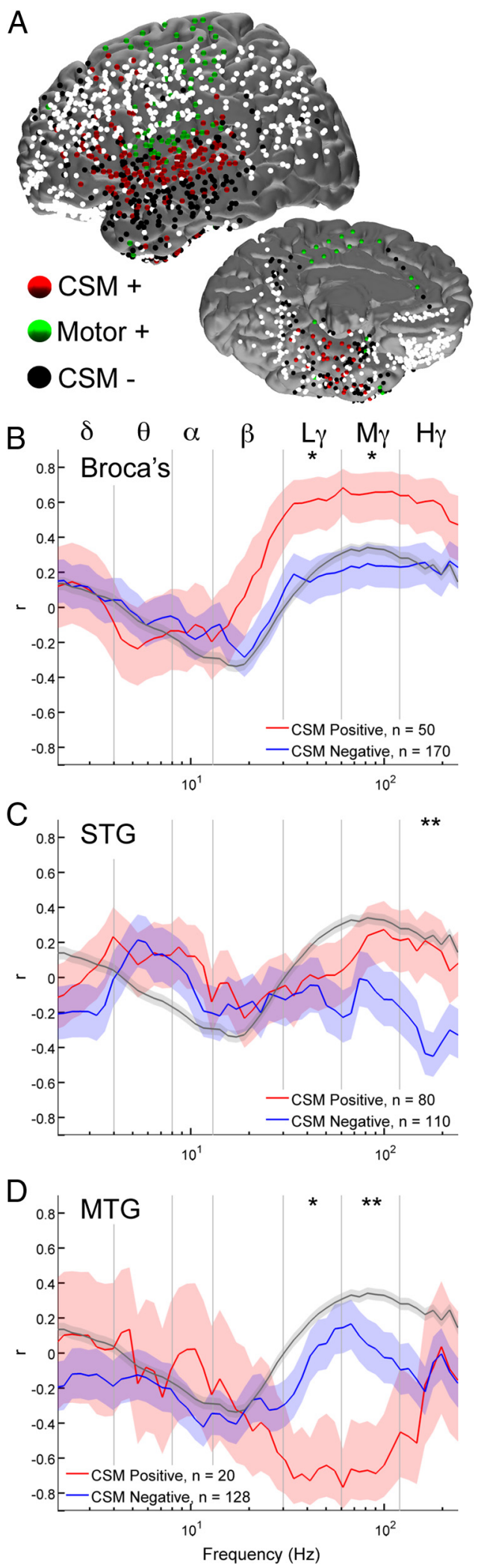

Figure 7. LFP-BOLD coupling for regions determined as essential for language by cortical stimulation mapping, CSM. A, Electrodes were transformed to a common surface and then color coded based on the CSM results. Electrodes were classified as CSM positive (red), negative (black), or motor only (green). Electrodes that were not tested are displayed as white. $\boldsymbol{B}-\boldsymbol{D}$, Sites that were CSM positive for receptive or expressive language (not just visual naming) were located in Broca's area $(n=25)(B)$, STG $(n=40)(C)$, and MTG $(D)(n=10)$. LBC curves of CSM-positive versus negative sites in these three regions were compared. The shaded error bars depict an uncorrected $p<0.05$ and the gray line represents all other electrodes used in the (a number that increases to $28 \%$ when the lobar location is considered). The heuristic of "low-frequency, negative correlation; high-frequency, positive correlation" (Kilner et al., 2005) is consistent with these data. Our data show important distinctions in the LBC function related to the cortical locus of interest (Figs. 4, 5 ), which should be considered when making comparisons of fMRI activity across brain regions.

Consistent with prior reports, we found that the biggest contributor to the LBC during cognitive processes is gamma band activity (Logothetis et al., 2001). While univariate regressions of activity in each band against the BOLD signal (Figs. 3-5) show many significant interactions, only beta and mid-gamma bands are significant in a multivariate analysis (Ojemann et al., 2010). This suggests multicolinearity within low (theta, alpha, and beta) and within high frequencies (low, mid, and high gamma). We note that the component of the gamma band that correlated best with fMRI was mid-gamma $(60-120 \mathrm{~Hz})$ (Fig. 3). This is consistent with other such correlations during human cognition (Lachaux et al., 2007; Dalal et al., 2009; Ojemann et al., 2010; Khursheed et al., 2011), but differs from observations in simian $\mathrm{V} 1$, where the best correlation was in the low-gamma band (Goense and Logothetis, 2008).

We also found that activity in mid-gamma and beta bands are poorly intercorrelated $\left(r^{2}=0.03\right)$. Beta band activity is therefore an independent contributor to the observed BOLD signal change. Other human experiments, during cognitive control and visual discrimination, also reveal an inverse relationship of beta band activity with the fMRI signal (Swann et al., 2009; Ojemann et al., 2010; Scheeringa et al., 2011). Beta band activity likely mediates top-down cognitive control and maintenance of the status quo (Engel and Fries, 2010). Decrease in beta reflects new, extrinsic input into a system that transitions from the default mode to an "activation" state (Dalal et al., 2009; van Elk et al., 2010). Measuring these effects coincides with the intended goals of fMRI, and in this context beta decreases do correspond with a BOLD signal increase. Spatial variations in the LBC function could then be driven by differences in correlation between LFP and BOLD signals in either beta or gamma bands (Figs. 5, 6).

The ability to make estimates at widely dispersed locations is crucial to measuring spatial variability in the LBC function (Mukamel et al., 2005; Martuzzi et al., 2009; Scheeringa et al., 2009). LFPs are a more direct measure of neural activity than the hemodynamic response. While not a perfect estimate of aggregate processing in a cortical module, they are a summation of perisynaptic activity (Logothetis et al., 2001; Goense and Logothetis, 2008; Ekstrom et al., 2009) and integrate both long-range input with local small-scale reentrant processes (Angenstein et al., 2009). Concurrent optogenetic-LFP recordings suggest that different frequency bands originate from distinct cell populations. Large pyramidal cells produce lower frequencies (beta), and fastspiking interneurons contribute to faster oscillations (gamma) (Cardin et al., 2009). Given the finding here and elsewhere (Scheeringa et al., 2011) that beta and gamma contribute separately to the hemodynamic response, nuanced approaches are needed to interpret the BOLD signal as broadly equivalent across brain regions. Activation may not imply roughly equivalent underlying neural activity.

\section{$\leftarrow$}

analysis (see Fig. 3B). Significant differences were noted between CSM-positive and CSMnegative sites in the gamma band correlation for all three gyri $\left({ }^{*} p<0.01,{ }^{* *} p<0.001\right)$. 

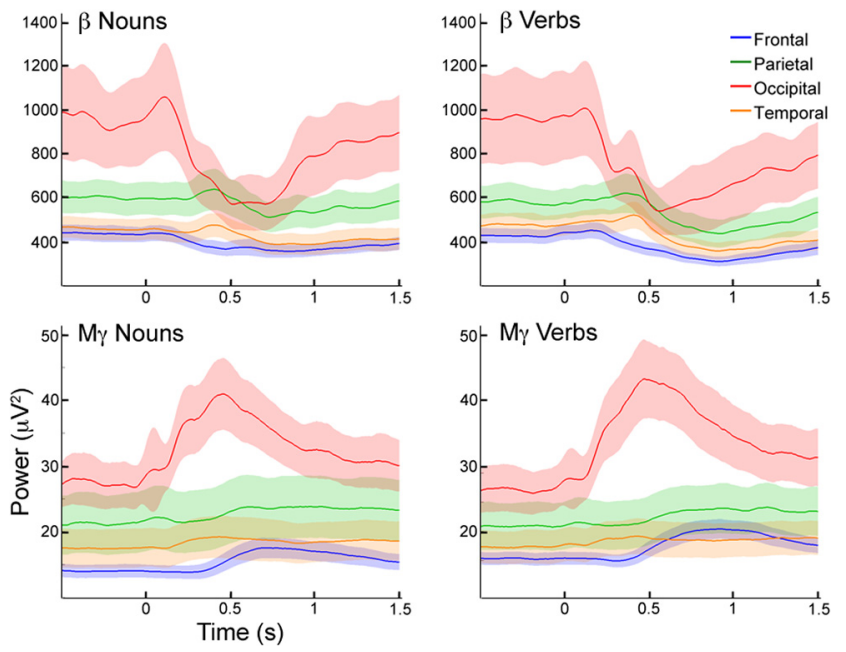

Figure 8. Changes in $\mathrm{ECOG}$ power from baseline during task performance. For each lobe, the time course of beta and mid-gamma $(M \gamma)$ power at each electrode was computed from $500 \mathrm{~ms}$ before stimulus onset to $1500 \mathrm{~ms}$ after. The average and $95 \% \mathrm{Cl}$ are plotted for all electrodes in each lobe. Task related attenuation in beta power was noted in both tasks after stimulus onset, concurrent with an increase in power in the mid-gamma band. Resting power in both bands was greatest in the occipital lobe and lowest in frontal cortex.

Given the spatial variability in the cortical microarchitecture, we expected to find interregional variability in the LBC function (Logothetis, 2008). fMRI after electrical stimulation of the sensorimotor cortex in rats (Sloan et al., 2010) has revealed variations in neurovascular coupling between cortical and subcortical regions. Our work extends this finding into cognitive processes in humans, a subject of interest to functional neuroimaging. Differences in the LBC function between lobes became more manifest as electrodes were further subcategorized based on gyral location (Fig. 6). Substantial deviation from the mean was noted across frequency bands, but especially in beta and mid-gamma bands. Gamma band ECoG activity in the lateral temporal neocortex (STG, MTG) and the parietal lobe (PoCG) showed weak correlations with the fMRI signal.

One explanation of the regional differences in LBC may be the degree of activity in a region in the "resting state." Components of the default mode network (Raichle et al., 2001; Laufs et al., 2003) with greater beta power during rest may show greater desynchronization in beta during activation. This may explain why frontal electrodes (most of which were lateral frontal) show a lower degree of beta decrease for a given fMRI signal change (Figs. 4, 5). Studies of the default mode network (Dastjerdi et al., 2011) evaluated contrasts in task-related gamma band activity but have not commented on resting ECoG power. To assess regional distinctions in the resting ECoG and task-induced modulation, we analyzed $500 \mathrm{~ms}$ of time before stimulus onset to evaluate the evolution of changes in beta and mid-gamma during noun and verb generation (Fig. 8). Baseline beta and gamma power were greatest in occipital and parietal lobes and least in the frontal lobe. The greater baseline beta power in posterior regions affords the possibility of larger attenuation compared to frontal regions. Conversely, the low amount of mid-gamma activity in frontal regions allows for a greater percentage change in power during activation. Thus, resting power in each brain region could impact the magnitude (regression coefficient) of the LBC.

Another explanation for spatial distinctions in the LBC function may lie in differences in the degree to which language function is distributed in different brain regions (Logothetis, 2008).
Temporal lobe areas may exhibit a greater degree of distributed processing of language than frontal lobe areas. This may be why prior comparisons of LBC in humans during visual, auditory, and sensorimotor processing (Mukamel et al., 2005; Lachaux et al., 2007; Scheeringa et al., 2011) showed little variation in the LBC. Spatial variations in the LBC function may also explain the failure of fMRI in reliably localizing ELS yet successfully localizing sensorimotor and visual cortices (Rutten et al., 2002; Roux et al., 2003).

A critique of data collected from patients is that it reflects a population whose physiology varies from the norm. All patients included here are fully independent individuals with focal epilepsy. All except one had an IQ in the average range (Table 1) and were able to perform language and memory tasks without difficulty with response times in normative ranges. To account for any confounds related to pathologic tissue, electrodes overlying sites of IEDs and regions involved with seizure onsets were systematically removed from the analysis, as were any trials where IEDs were noted at any locus. A power analysis to determine the number of trials necessary to reach a given confidence level in the $t$ value of the contrast between naming and scrambled conditions revealed that for our paradigm and recording environment, approximately 40 trials were needed. All but one of our patients had $>40$ trials in each condition.

An important consideration in such comparisons is to minimize variations between tasks in the two experimental conditions. While concurrent ECoG-fMRI (Vulliemoz et al., 2011) is technically feasible, multiregional sampling with metallic macroelectrodes produces substantial susceptibility artifact and degrades BOLD signal. Additionally, there is risk of thermal injury due to heating of electrodes by currents induced by fluctuating EPI gradients. We matched our two experimental conditions as closely as possible to each other. The disparate effects of overt articulation during ECoG and button presses during fMRI were minimized with the use of the control condition (scrambled images). During fMRI patients pressed a button to respond to scrambled images, while in the epilepsy monitoring unit they said the word "scrambled." In the grouped fMRI analysis (Fig. 2), there is no significant activation in primary hand motor cortex in the fMRI condition. Additionally, restricting the ECoG analysis to a window that ends at 1SD before articulation minimizes the potential effects of differences in the articulation of a verb/noun versus saying "scrambled." Robust differences between LBC estimates in regions not expected to relate to task differences-e.g., orbitofrontal cortex and MTG (Fig. 6) provide validation for our thesis. Despite our controls, some of the differences between tasks may have had subtle effects on the estimate of the LBC function. This could be minimized in future work by using event-related fMRI design with overt articulation in the scanner. This will also minimize contribution of global state or practice effects resulting from slight differences between ECoG design (where scrambled trials were interspersed with naming trials) and block design fMRI. Furthermore, the two naming tasks (Shapiro et al., 2006) involve distinct but overlapping substrates. Future work will address task-dependent variations in LBC using varied language tasks, such as those that depend on auditory rather than visual cues.

Both the general linear model and mixed effects model proposed here explain approximately a third of the variance in the BOLD signal. Intrinsic physiologic differences between these two measures, the limited sampling of sulcal sources by the ECoG, the empiric assumption of a linear relationship between measures, and relatively poor signal-to-noise characteristics of the BOLD 
signal (compared with ECoG) may all account for this less than perfect covariance. There remain important questions to be asked of actual (rather than contrast-dependent) decreases in BOLD. Further work will include alternate modeling methods including Bayesian analysis, as well as specific evaluation of "negative" BOLD responses.

\section{References}

Angenstein F, Kammerer E, Scheich H (2009) The BOLD response in the rat hippocampus depends rather on local processing of signals than on the input or output activity. A combined functional MRI and electrophysiological study. J Neurosci 29:2428-2439.

Brown EC, Rothermel R, Nishida M, Juhász C, Muzik O, Hoechstetter K, Sood S, Chugani HT, Asano E (2008) In vivo animation of auditorylanguage-induced gamma-oscillations in children with intractable focal epilepsy. Neuroimage 41:1120-1131.

Bruns A (2004) Fourier-, Hilbert- and wavelet-based signal analysis: are they really different approaches? J Neurosci Methods 137:321-332.

Cardin JA, Carlén M, Meletis K, Knoblich U, Zhang F, Deisseroth K, Tsai LH, Moore CI (2009) Driving fast-spiking cells induces gamma rhythm and controls sensory responses. Nature 459:663-667.

Cohen J, Cohen P (1983) Applied multiple regression/correlation analysis for the behavioral sciences, Ed 2. Hillsdale, NJ: Lawrence Erlbaum.

Cox RW (1996) AFNI: software for analysis and visualization of functional magnetic resonance neuroimages. Comput Biomed Res 29:162-173.

Crone NE, Hao L, Hart J Jr, Boatman D, Lesser RP, Irizarry R, Gordon B (2001) Electrocorticographic gamma activity during word production in spoken and sign language. Neurology 57:2045-2053.

Dalal SS, Baillet S, Adam C, Ducorps A, Schwartz D, Jerbi K, Bertrand O, Garnero L, Martinerie J, Lachaux JP (2009) Simultaneous MEG and intracranial EEG recordings during attentive reading. Neuroimage 45:1289-1304.

Dale AM, Fischl B, Sereno MI (1999) Cortical surface-based analysis. I. Segmentation and surface reconstruction. Neuroimage 9:179-194.

Dastjerdi M, Foster BL, Nasrullah S, Rauschecker AM, Dougherty RF, Townsend JD, Chang C, Greicius MD, Menon V, Kennedy DP, Parvizi J (2011) Differential electrophysiological response during rest, selfreferential, and non-self-referential tasks in human posteromedial cortex. Proc Natl Acad Sci U S A 108:3023-3028.

Ekstrom A (2010) How and when the fMRI BOLD signal relates to underlying neural activity: the danger in dissociation. Brain Res Rev 62:233-244.

Ekstrom A, Suthana N, Millett D, Fried I, Bookheimer S (2009) Correlation between BOLD fMRI and theta-band local field potentials in the human hippocampal area. J Neurophysiol 101:2668-2678.

Ellis AW, Burani C, Izura C, Bromiley A, Venneri A (2006) Traces of vocabulary acquisition in the brain: Evidence from covert object naming. Neuroimage 33:958-968.

Ellmore TM, Beauchamp MS, Breier JI, Slater JD, Kalamangalam GP, O'Neill TJ, Disano MA, Tandon N (2010) Temporal lobe white matter asymmetry and language laterality in epilepsy patients. Neuroimage 49:2033-2044.

Engel AK, Fries P (2010) Beta-band oscillations-signalling the status quo? Curr Opin Neurobiol 20:156-165.

Goense JB, Logothetis NK (2008) Neurophysiology of the BOLD fMRI signal in awake monkeys. Curr Biol 18:631-640.

Hamberger MJ, Seidel WT, McKhann GM 2nd, Perrine K, Goodman RR (2005) Brain stimulation reveals critical auditory naming cortex. Brain 128:2742-2749.

Indefrey P, Levelt WJ (2004) The spatial and temporal signatures of word production components. Cognition 92:101-144.

Indefrey P, Kleinschmidt A, Merboldt KD, Krüger G, Brown C, Hagoort P, Frahm J (1997) Equivalent responses to lexical and nonlexical visual stimuli in occipital cortex: a functional magnetic resonance imaging study. Neuroimage 5:78-81.

Khursheed F, Tandon N, Tertel K, Pieters TA, Disano MA, Ellmore TM (2011) Frequency-specific electrocorticographic correlates of working memory delay period fMRI activity. Neuroimage 56:1773-1782.

Kilner JM, Mattout J, Henson R, Friston KJ (2005) Hemodynamic correlates of EEG: a heuristic. Neuroimage 28:280-286.

Lachaux JP, Rudrauf D, Kahane P (2003) Intracranial EEG and human brain mapping. J Physiol Paris 97:613-628.
Lachaux JP, Fonlupt P, Kahane P, Minotti L, Hoffmann D, Bertrand O, Baciu M (2007) Relationship between task-related gamma oscillations and BOLD signal: new insights from combined fMRI and intracranial EEG. Hum Brain Mapp 28:1368-1375.

Laufs H, Krakow K, Sterzer P, Eger E, Beyerle A, Salek-Haddadi A, Kleinschmidt A (2003) Electroencephalographic signatures of attentional and cognitive default modes in spontaneous brain activity fluctuations at rest. Proc Natl Acad Sci U S A 100:11053-11058.

Le Van Quyen M, Foucher J, Lachaux J, Rodriguez E, Lutz A, Martinerie J, Varela FJ (2001) Comparison of Hilbert transform and wavelet methods for the analysis of neuronal synchrony. J Neurosci Methods 111:83-98.

Liljeström M, Hultén A, Parkkonen L, Salmelin R (2009) Comparing MEG and fMRI views to naming actions and objects. Hum Brain Mapp 30:1845-1856.

Logothetis NK (2008) What we can do and what we cannot do with fMRI. Nature 453:869-878.

Logothetis NK, Pauls J, Augath M, Trinath T, Oeltermann A (2001) Neurophysiological investigation of the basis of the fMRI signal. Nature 412:150-157.

Martuzzi R, Murray MM, Meuli RA, Thiran JP, Maeder PP, Michel CM, Grave de Peralta Menendez R, Gonzalez Andino SL (2009) Methods for determining frequency- and region-dependent relationships between estimated LFPs and BOLD responses in humans. J Neurophysiol 101:491-502.

Mukamel R, Gelbard H, Arieli A, Hasson U, Fried I, Malach R (2005) Coupling between neuronal firing, field potentials, and FMRI in human auditory cortex. Science 309:951-954.

Nagasawa T, Juhasz C, Rothermel R, Hoechstetter K, Sood S, Asano E (2011) Spontaneous and visually driven high-frequency oscillations in the occipital cortex: intracranial recording in epileptic patients. Hum Brain Mapp. Advance online publication. Retrieved March 22, 2011. doi:10.1002/hbm.21233.

Ojemann GA, Corina DP, Corrigan N, Schoenfield-McNeill J, Poliakov A, Zamora L, Zanos S (2010) Neuronal correlates of functional magnetic resonance imaging in human temporal cortex. Brain 133:46-59.

Paternoster R, Brame R, Mazerolle P, Piquero A (1998) Using the correct statistical test for the equality of regression coefficients. Criminology 36:859-866.

Price CJ (2000) The anatomy of language: contributions from functional neuroimaging. J Anat 197:335-359.

Price CJ, Friston KJ (1999) Scanning patients with tasks they can perform. Hum Brain Mapp 8:102-108.

Price CJ, Moore CJ, Humphreys GW, Frackowiak RS, Friston KJ (1996) The neural regions sustaining object recognition and naming. Proc Biol Sci 263:1501-1507.

Raichle ME, MacLeod AM, Snyder AZ, Powers WJ, Gusnard DA, Shulman GL (2001) A default mode of brain function. Proc Natl Acad Sci U S A 98:676-682.

Roux FE, Boulanouar K, Lotterie JA, Mejdoubi M, LeSage JP, Berry I (2003) Language functional magnetic resonance imaging in preoperative assessment of language areas: correlation with direct cortical stimulation. Neurosurgery 52:1335-1345; discussion 1345-1347.

Rutten GJ, Ramsey NF, van Rijen PC, Noordmans HJ, van Veelen CW (2002) Development of a functional magnetic resonance imaging protocol for intraoperative localization of critical temporoparietal language areas. Ann Neurol 51:350-360.

Saad ZS, Ropella KM, DeYoe EA, Bandettini PA (2003) The spatial extent of the BOLD response. Neuroimage 19:132-144.

Salmelin R, Hari R, Lounasmaa OV, Sams M (1994) Dynamics of brain activation during picture naming. Nature 368:463-465.

Scheeringa R, Petersson KM, Oostenveld R, Norris DG, Hagoort P, Bastiaansen MC (2009) Trial-by-trial coupling between EEG and BOLD identifies networks related to alpha and theta EEG power increases during working memory maintenance. Neuroimage 44:1224-1238.

Scheeringa R, Fries P, Petersson KM, Oostenveld R, Grothe I, Norris DG, Hagoort P, Bastiaansen MC (2011) Neuronal dynamics underlying high- and low-frequency EEG oscillations contribute independently to the human BOLD signal. Neuron 69:572-583.

Shapiro KA, Moo LR, Caramazza A (2006) Cortical signatures of noun and verb production. Proc Natl Acad Sci U S A 103:1644-1649.

Sloan HL, Austin VC, Blamire AM, Schnupp JW, Lowe AS, Allers KA, Matthews PM, Sibson NR (2010) Regional differences in neurovascular 
coupling in rat brain as determined by fMRI and electrophysiology. Neuroimage 53:399-411.

Specht K, Huber W, Willmes K, Shah NJ, Jäncke L (2008) Tracing the ventral stream for auditory speech processing in the temporal lobe by using a combined time series and independent component analysis. Neurosci Lett 442:180-185.

Swann N, Tandon N, Canolty R, Ellmore TM, McEvoy LK, Dreyer S, DiSano M, Aron AR (2009) Intracranial EEG reveals a time- and frequencyspecific role for the right inferior frontal gyrus and primary motor cortex in stopping initiated responses. J Neurosci 29:12675-12685.

Szekely A, D’Amico S, Devescovi A, Federmeier K, Herron D, Iyer G, Jacobsen T, Arévalo AL, Vargha A, Bates E (2005) Timed action and object naming. Cortex 41:7-25.

Tandon N (2008) Cortical mapping by electrical stimulation of subdural electrodes: language areas. In: Textbook of epilepsy surgery (Luders HO, ed), pp 1001-1015. New York: Informa HealthCare.
Tertel K, Tandon N, Ellmore TM (2010) Probing brain connectivity by combined analysis of diffusion MRI tractography and electrocorticography. Comput Biol Med. Advance online publication. Retrieved December 3, 2010. doi:10.1016/j.compbiomed.2010.11.004.

van Elk M, van Schie HT, Zwaan RA, Bekkering H (2010) The functional role of motor activation in language processing: motor cortical oscillations support lexical-semantic retrieval. Neuroimage 50:665-677.

Vulliemoz S, Carmichael DW, Rosenkranz K, Diehl B, Rodionov R, Walker MC, McEvoy AW, Lemieux L (2011) Simultaneous intracranial EEG and fMRI of interictal epileptic discharges in humans. Neuroimage 54:182-190.

Wada J, Rasmussen T (2007) Intracarotid injection of sodium amytal for the lateralization of cerebral speech dominance. 1960. J Neurosurg 106:1117-1133.

Xue G, Dong Q, Chen C, Lu Z, Mumford JA, Poldrack RA (2010) Greater neural pattern similarity across repetitions is associated with better memory. Science 330:97-101. 